\title{
Circular RNA circSPATA6 Inhibits the Progression of Oral Squamous Cell Carcinoma Cells by Regulating TRAF6 via miR-I82
}

This article was published in the following Dove Press journal: Cancer Management and Research

\section{Xinhua Fan \\ Ying Wang}

Department of Stomatology, Inner Mongolia Baotou Steel Hospital, Baotou City, Inner Mongolia, People's Republic of China
Correspondence: Ying Wang

Tel +86-472-5992490

Email wybaotou0812@I26.com
Background: Oral squamous cell carcinoma (OSCC) has become a widely concerned social problem. Circular RNA spermatogenesis-associated protein 6 (circSPATA6) exhibited low expression in OSCC tissues, yet the regulatory mechanism of circSPATA6 remains vague.

Methods: Levels of circSPATA6, linear SPATA6, microRNA-182 (miR-182), and tumor necrosis factor receptor-associated factor 6 (TRAF6) were detected by real-time quantitative polymerase chain reaction (RT-qPCR). Migration, invasion, cell cycle arrest, and apoptosis were assessed by Wound-healing, Matrigel invasion, and Flow cytometry assays. The binding relationship between miR-182 and circSPATA6 or TRAF6 was predicted by circRNA interactome or DIANA TOOL and then proved by the dual-luciferase reporter and RNA Immunoprecipitation (RIP) assays. TRAF6 protein level was measured by Western blot assay. The biological role of circSPATA6 on OSCC tumor growth was analyzed by xenograft tumor model in vivo. Exosomes were isolated and detected by differential centrifugation and a transmission electron microscope.

Results: CircSPATA6 and TRAF6 were declined, and miR-182 was elevated in OSCC cells. Functionally, circSPATA6 impeded migration and invasion, and facilitated cell cycle arrest and apoptosis of OSCC cells. Mechanistically, circSPATA6 could modulate TRAF6 expression through sponging miR-182. Moreover, circSPATA6 blocked tumor growth in the OSCC mice model. Exosomal circSPATA6 retarded the growth of OSCC cells.

Conclusion: CircSPATA6 curbed migration and invasion, and expedited cell cycle arrest and apoptosis in OSCC cells partly through regulating the miR-182/TRAF6 axis. These findings hinted at an underlying circRNA-targeted therapy for OSCC.

Keywords: circSPATA6, miR-182, TRAF6, oral squamous cell carcinoma

\section{Introduction}

Oral squamous cell carcinoma (OSCC), a type of head and neck cancer, has gradually become a widely concerned social problem due to the highly metastatic and invasive malignancy. ${ }^{1,2}$ Although the diagnostic and therapeutic strategies have improved, the survival rate of the general OSCC patients remains unsatisfactory. ${ }^{3}$ Hence, to develop more effective therapies for OSCC, it is important to explore new targets and potential molecular mechanisms.

Recently, as a new class of non-coding RNAs, circular RNAs (circRNAs) have increasingly attracted emphasis due to the special structure, a covalently closed loop neither a $5^{\prime}$ cap nor a 3'-polyadenylated tail. ${ }^{4,5}$ Compared with linear counterparts, they are abundant and stable in plasma and tissues and might be potential 
biomarkers for disease diagnosis and prognosis, including cancer. $^{6,7}$ Ubiquitous in plasma, exosomes, tissues and spatiotemporal specificity, they could regulate gene expression in various levels. ${ }^{8-10}$ Emerging evidence indicates that circRNAs have been reported as key mediators of some tumors' progression. In fact, as carcinogens or tumor suppressors, circRNAs were identified to take part in the modulation of OSCC development. Ouyang et al indicated that the overexpression of hsa_circ_0109291 intensified the malignancy of OSCC through boosting proliferation and migration. ${ }^{11}$ Gao et al described that circPKD2 dampened growth and metastasis through the miR204-3p/APC2 axis in OSCC. ${ }^{12}$ Interestingly, a novel circRNA, circSPATA6 (hsa_circ_0008202) is derived from exons of spermatogenesis-associated protein 6 (SPATA6). Moreover, it has been confirmed that circSPATA6 was downregulated in OSCC tissues, implying that circSPATA6 might be involved in OSCC progression. $^{13}$ However, the underlying molecular mechanism of circSPATA6 on OSCC has not been fully elucidated.

Over the last decade, another kind of small, endogenous, non-coding RNA, microRNAs (miRNAs) has been reported to exert biological function through the interaction with the 3 'UTR of target mRNAs. ${ }^{14}$ Numerous miRNAs are known to play vital roles in the regulation of various biological and cellular activities, including triggering or inhibiting tumor progression. ${ }^{15-18}$ MicroRNA182 (miR-182), as a carcinogenic agent, has been proved in a variety of tumors. ${ }^{19-21}$ It was previously reported that miR-182 showed a high expression in head and neck squamous cell carcinoma. $^{22}$ Some documentation indicated that miR-182 expedited OSCC cell growth and metastasis by regulating target genes. ${ }^{23,24}$ These data suggested the vital role of miR-182 in OSCC development.

As a kind of adaptor protein and E3 ubiquitin ligase, tumor necrosis factor receptor-associated factor 6 (TRAF6) has been confirmed to activate diverse signaling pathways, such as the NF- $\kappa \mathrm{B}$ signaling pathway ${ }^{25}$ and JAK-STAT signaling pathway. ${ }^{26}$ Some studies have exhibited that the low expression of TRAF6 in cervical cancer ${ }^{27}$ and colorectal cancer. ${ }^{28}$ Furthermore, related studies manifested that the knockdown of TRAF6 could reinforce the oncogenic phenotypes in OSCC cells, ${ }^{29,30}$ indicating that TRAF6 might be acting as a tumor suppressor in OSCC.

Herein, our data presented that circSPATA6 hindered migration and invasion, and accelerated apoptosis rate and cell cycle arrest in OSCC cells. Using bioinformatics analysis, we found that there were some latent binding sites between miR-182 and circSPATA6 or TRAF6. Therefore, this study was designed to identify whether the regulatory role of the circSPATA6 was mediated through the miR-182/TRAF6 axis in OSCC.

\section{Materials and Methods Clinical Samples and Cell Culture}

Samples of serum from OSCC patients $(n=46)$ and healthy volunteers $(n=25)$ were acquired from Inner Mongolia Baotou Steel Hospital. This research got the approval of the Ethics Committee of Inner Mongolia Baotou Steel Hospital, and written informed consent was signed from every participant. All methods were conducted in accordance with the relevant guidelines and regulations and the Declaration of Helsinki.

The human OSCC cell lines (CAL-27 and SCC9) were provided by American Type Culture Collection (ATCC, Manassas, VA, USA) and OSCC cell line HSC6 was obtained from Shanghai Honsun Biological Technology Co., Ltd (Shanghai, China), and OSCC cell line UM1 was purchased by Japanese Collection of Research Bioresources (JCRB, Osaka, Japan). Human oral keratinocyte (HOK) cells were acquired by Cell Bank of the Chinese Academy of Sciences (Shanghai, China), and maintained in Keratinocyte-SFM medium (Invitrogen, Carlsbad, CA, USA). OSCC cells were cultured in Dulbecco's modified Eagle's medium (DMEM; Gibco, Carlsbad, CA, USA) with 10\% fetal bovine serum (FBS; Invitrogen) and 1\% penicillin/streptomycin (KeyGen, Nanjing, China). All cells were maintained in a humidified atmosphere containing $5 \% \mathrm{CO}_{2}$ at $37^{\circ} \mathrm{C}$.

\section{Real-Time Quantitative Polymerase Chain Reaction (RT-qPCR)}

According to the user's guidebook of RNAiso Plus (TaKaRa, Dalian, China), total RNA from tissues and cells were isolated. Subsequently, the extracted RNA was reverse-transcribed to the first-strand complementary DNA (cDNA) using the PrimeScript RT Reagent Kit (TaKaRa) and microRNA First-Strand cDNA Synthesis Kit (Fulen Gene, Guangzhou, China). On an ABI PRISM7500 Sequence Detection System (Applied Biosystems, Foster City, CA, USA), RT-qPCR was conducted in line with SYBR Green PCR Kit (Takara). The $2^{-\Delta \Delta C t}$ method was used to calculate the obtained data, with glyceraldehyde 3-phosphate dehydrogenase (GAPDH for circSPATA6 and 
TRAF6) and U6 (for miR-182) as the internal reference. The primer sequences are presented in Table 1 .

\section{RNase R Treatment}

Total RNA was incubated with or without RNase R (3 U/ $\mathrm{mg}$, Epicentre, Shanghai, China) for $15 \mathrm{~min}$ at $37^{\circ} \mathrm{C}$, followed by purification with phenol-chloroform (Sigma, St. Louis, Mo, USA). And then, the expression level of circular and linear SPATA6 was examined using RT-qPCR.

\section{Cell Transfection}

CircSPATA6 overexpression vector was achieved through inserting the circSPATA6 sequence into the empty pcDNA vector (Invitrogen, as a control). MiR-182 mimic (miR-182), miR-182 inhibitor (in-miR-182), circSPATA6 small interference RNA (si-circSPATA6), si-TRAF6, and their negative controls (miR-con, in-miR-con, si-con), were designed and synthesized from Ribobio (Guangzhou, China) and GenePharma (shanghai, China). According to the supplier's direction of Lipofectamine 3000 (Invitrogen), CAL-27 and HSC6 cells were transfected with the above vectors and oligonucleotides. After $48 \mathrm{~h}$ incubation, the transfection efficiency of cells was examined using RT-qPCR assay.

\section{Wound Healing Assay}

In this assay, transfected CAL-27 HSC6 cells in 6-well plates were trypsinized and reseeded. After scratching with

Table I The Sequences of Primers for RT-PCR Used in This Study

\begin{tabular}{|l|l|}
\hline Names & Sequences (5'-3') \\
\hline CircSPATA6: Forward & AAGGTCGTGTAGTAAGCCCC \\
\hline CircSPATA6: Reverse & TGTTGAGGGTGCTGAATGGA \\
\hline SPATA6: Forward & AAGGGAAAATCCCACCGACC \\
\hline SPATA6: Reverse & TGTACAAGAAAGTTGGGCAGA \\
\hline MiR-182: Forward & GGCAATGGTAGAACTCAC \\
\hline MiR-I82: Reverse & GAACATGTCTGCGTATCTC \\
\hline TRAF6: Forward & GCCTGGGTGACAGAGTGC \\
\hline TRAF6: Reverse & AATGACTACTTATGGCTCCTTTTC \\
\hline U6: Forward & CTCGCTTCGGCAGCACA \\
\hline U6: Reverse & AACGCTTCACGAATTTGCGT \\
\hline GAPDH: Forward & GCACCGTCAAGGCTGAGAAC \\
\hline GAPDH: Reverse & TGGTGAAGACGCCAGTGGA \\
\hline
\end{tabular}

a $200 \mu \mathrm{L}$ sterile pipette tip (record $0 \mathrm{~h}$ ), the cells were incubated with serum-free medium for $24 \mathrm{~h}$, and then the detached cells were removed with phosphate-buffered saline (PBS, Invitrogen). At last, the migration rate was detected using an inverted microscope (Olympus, Tokyo, Japan) (magnification $\times 100$ ), and the distance of $0-24$ $\mathrm{h}$ was measured with an Image pro plus 6.0 software (Media Cybernetics, Rockville, MD, USA).

\section{Cell Invasion Assay}

After collected and re-suspended in medium with $1 \%$ FBS, transfected CAL-27 and HSC6 cells were seeded in the upper compartment with matrigel (BD Bioscience, San Jose, CA, USA), and $20 \%$ FBS as a chemoattractant was added into the lower chamber, followed by incubation for 24 h. And then, a cotton swab was applied to scrape the cells on the upper chamber, and $0.1 \%$ crystal violet (Sigma-Aldrich, St. Louis, MO, USA) was adopted to stain the cells invaded into the lower surface. Finally, the photographing and counting of the stained cells were carried out using an inverted microscope (Olympus) (magnification $\times 100$ ).

\section{Cell Apoptosis and Cell Cycle Assays}

In brief, transfected cells were harvested, and washed with PBS (Invitrogen), followed by fixation with ethanol for 1 h. After measurement with FACSan flow cytometry (BD Biosciences) with $5 \mu \mathrm{L}$ Annexin (V-fluorescein isothiocyanate) V-FITC/Propidium Iodide (PI) (Beyotime, Nantong, China), apoptosis cells were analyzed with Quest software (BD Biosciences). For cell cycle assay, after treatment with ethanol and PBS (Invitrogen), cells were added to RNaseA and PI for $30 \mathrm{~min}$ at room temperature. Finally, FACSan flow cytometry (BD Biosciences) and ModFit LT 3.2 software (Verity Software House, Topsham, ME, USA) were utilized to analyze the percentages of cells in the G0-G1 and S phases.

\section{Dual-Luciferase Reporter Assay}

Generally, the sequences of circSPATA6 and TRAF6 3'UTR possessing wild-type (WT) or mutant-type (MUT) miR-182 binding sites were cloned into psiCHECK-2 vector (Promega, Madison, WI, USA), generating circSPATA6-WT/MUT, and TRAF6-WT/MUT 3'UTR reporter plasmids. According to the Lipofectamine 3000 (Invitrogen), the above plasmids were transfected into CAL-27 and HSC6 cells together with Blank, miR-con, or miR-182. After $48 \mathrm{~h}$ of transfection, the luciferase activities were measured with a dual-luciferase reporter assay kit (Promega) and normalized to Renilla activity. 


\section{RNA Immunoprecipitation (RIP)}

In this assay, RIP assay was performed according to the supplier's direction of Magna RIP RNA-Binding Protein Immunoprecipitation Kit (Millipore, Billerica, MA, USA). After being lysed in the complete RIP lysis buffer at $80 \%$ confluence, CAL-27 and HSC6 cell extracts were incubated with magnetic beads conjugated with anti-Argonaute 2 antibody (Anti-Ago2; Millipore) or negative control normal mouse IgG (Millipore). After $24 \mathrm{~h}$ of incubation, samples were treated with proteinase $\mathrm{K}$, followed by purification and analysis using RT-qPCR assay.

\section{Western Blot Assay}

Total proteins were lysed by RIPA lysis buffer (Beyotime), followed by measurement with BCA Protein Assay Kit (Beyotime). After separation with a sodium dodecyl sulfate-polyacrylamide gel electrophoresis (SDS-PAGE) system, the protein sample (40 $\mu \mathrm{g})$ was further transferred to a nitrocellulose membrane (Millipore), followed by treatment with TBST containing 5\% non-fat milk. After $2 \mathrm{~h}$ of incubation, the membranes were hybridized with primary antibodies: TRAF6 (1:1000; ab33915, Abcam, Cambridge, UK) and $\beta$-actin $(1: 5000 ; \mathrm{ab} 8227, \mathrm{Abcam})$ at $4^{\circ} \mathrm{C}$. The next day, the membranes were probed with secondary antibody at room temperature for $1 \mathrm{~h}$. With the help of an ECL detection kit (Santa Cruz Biotechnology, Santa Cruz, CA, USA), the bands were detected, followed by analysis with Quantity One software (Bio-Rad, Hercules, CA, USA).

\section{Tumor Xenograft Assay}

The animal assay got the permission of the Animal Ethics Committee of the Inner Mongolia Baotou Steel Hospital, and was performed in compliances with the regulations for the Care and Use of Laboratory Animals and Guideline for Ethical Review of Animal (China, GB/T 35892-2018). First of all, Five-week-old male BALB/C nude mice (Vital River Laboratory, Beijing, China) were maintained in a specific pathogen-free (SPF) environment. And then, CAL-27 cells $\left(5 \times 10^{6}\right)$ transfected with Blank, pcDNA, and circSPATA6 were subcutaneously injected into the nude mice $(n=5$ per group). Tumor volumes were measured every week. At 4 weeks upon cell inoculation, the mice were sacrificed, and the tumors were collected, followed by weight and analysis with RT-qPCR and Western blot assays.

\section{Exosome Detection}

Exosomes from cells were isolated and purified as per the user's guidebook of ultracentrifugation and total exosome isolation kit (Thermo Fisher Scientific, Waltham, MA, USA). Whereafter, a transmission electron microscopy (HT7700, Hitachi, Tokyo, Japan) was employed to observe the exosomes on a fixative as described previously. ${ }^{31}$

\section{Statistical Analysis}

GraphPad Prism7 software was applied to analyze the obtained data. At least three independent data were documented as mean \pm standard deviation (SD), and a $P$-value $<0.05$ was considered significant. The differences between two or multiple groups were calculated using Student's $t$-test or oneway analysis of variance (ANOVA) with Tukey's tests.

\section{Results}

First of all, to investigate the function of circSPATA6 in OSCC, RT-qPCR assays were used for the detection of circSPATA6 expression. As presented in Figure 1A, compared with normal samples $(\mathrm{n}=25)$, a significant downregulation of circSPATA6 was observed in OSCC serum samples $(n=46)$. Furthermore, to determine the diagnostic value of circSPATA6 for OSCC, receiver operating characteristic (ROS) cure analysis was performed in serum samples. Results showed that the area under the ROS curve (AUC) of the utilization of circSPATA6 expression in serum samples for the diagnosis of OSCC was 0.7748 , implying the circSPATA6 is relatively closely associated with OSCC (Figure 1B). Moreover, we further validated that circSPATA6 was declined in OSCC cell lines (CAL-27, HSC6, UM1, and SCC9) versus Human oral keratinocyte (HOK) cells (Figure 1C). Especially in CAL-27 and HSC6 cells, so we chose these two cells for subsequent studies. These data suggested the involvement of circSPATA6 in OSCC progression. Also, to identify the stability of circSPATA6, CAL-27 and HSC6 cells were treated with RNase $\mathrm{R}^{+}$. Data exhibited that the treatment of RNase $\mathrm{R}$ apparently decreased the RNA level of Linear SPATA6, while had little effect on circSPATA6 level (Figure 1D and E). Meanwhile, since circRNAs do not have $3^{\prime}$ polyadenylated tail, we examined the existence of circSPATA6 in the reverse transcription products using Random primers or Oligo $(\mathrm{dT})_{18}$ primers. Data proved that circSPATA6 was almost undetectable when Oligo $(\mathrm{dT})_{18}$ primers were used in CAL-27 and HSC6 cells (Figure 1F and G), suggesting that circSPATA6 was a circular RNA. 

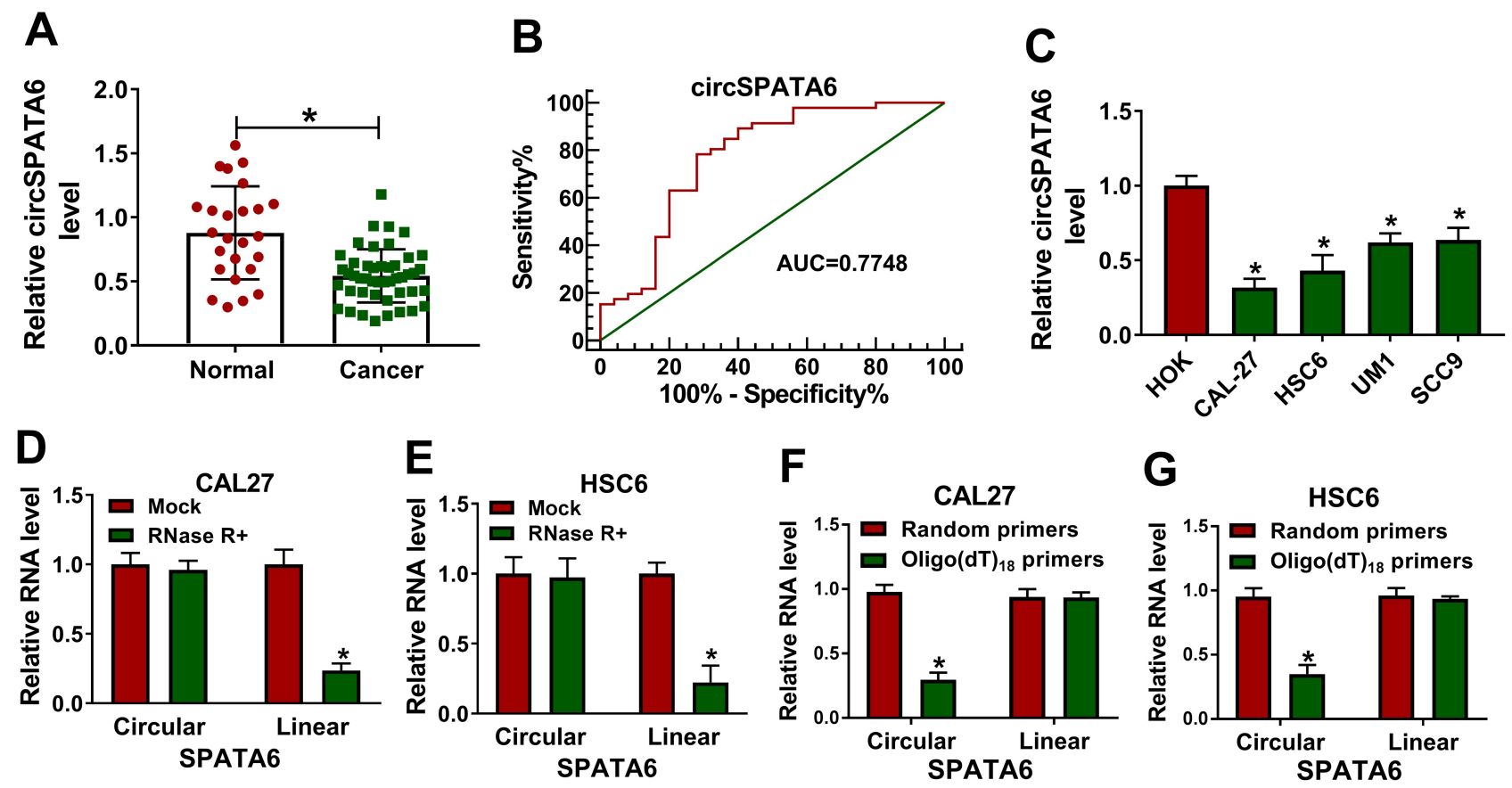

Figure I CircSPATA6 was downregulated in OSCC serum samples and cells. (A) RT-qPCR assay was employed to assess circSPATA6 in 46 OSCC serum samples and 25 normal samples. (B) Receiver operating characteristic (ROC) curve analysis revealed that circATRNLI was capable of distinguishing OSCC patients from healthy controls. Area under the ROC curve $(A \cup C)=0.7748,95 \%$, confidence interval $(\mathrm{Cl})=0.6496$ to 0.9 . (C) The expression level of circSPATA6 was detected in human oral keratinocyte (HOK) and OSCC cell lines (CAL-27, HSC6, UMI, and SCC9). (D and E) Relative RNA levels of circular SPATA6 and linear SPATA6 were tested in CAL-27 and HSC6 cells treated with or without RNase R $^{+}$. (F and $\mathbf{G}$ ) Relative RNA level of circular SPATA6 and linear SPATA6 were measured in CAL-27 and HSC6 cells by using Random primers or Oligo $(\mathrm{dT})_{18}$ primers. $* P<0.05$.

\section{CircSPATA6 Overexpression Repressed Migration, Invasion, Cell Cycle Progression, and Facilitated Apoptosis of OSCC Cells}

Then, to identify the role of circSPATA6 in OSCC cells, the over-expression plasmid of circSPATA6 was constructed. As displayed in Figure 2A, the expression level of circSPATA6 was strikingly increased in CAL-27 and HSC6 cells in comparison with blank control and empty vector control. Therefore, we utilized the gain-of-function system to further explore the role of circSPATA6, including, migration, invasion, cell cycle progression, and apoptosis. Data exhibited that overexpression of circSPATA6 hindered the abilities of migration and invasion of CAL-27 and HSC6 cells (Figure $2 \mathrm{~B}$ and $\mathrm{C}$ ). Subsequently, cell cycle analysis showed that the cells in the circSPATA6 group had an arrested cell cycle with a lower proportion of S-phase and a higher proportion of G0/ G1-phase relative to the control groups (Figure 2D). Meanwhile, flow cytometry assay was also used to detect the effect of circSPATA6 on cell apoptosis rate. As displayed in Figure 2E, compared with the blank control and empty vector control, the enhanced cell apoptosis was noticed on account of the upregulation of circSPATA6 in OSCC cells. All of these results indicated that the overexpression of circSPATA6 inhibited the progression of OSCC cells in vitro.

\section{CircSPATA6 Directly Bound with miR- 182}

It has been acknowledged that circRNAs could exert the function by acting as a molecular sponge for miRNAs. ${ }^{32}$ Thus, circular RNA interactome was applied to search the potential target miRNAs of circSPATA6. Results manifested that miR-182 has some complementary sequences with circSPATA6 (Figure 3A). Then, a dual-luciferase reporter assay was performed to verify the prediction. Data suggested that miR-182 mimics reduced the luciferase activity of circSPATA6-WT reporter vector, whereas had no marked impact on the luciferase activity of circSPATA6MUT reporter vector (Figure 3B and C). Meanwhile, to further confirm the direct binding between circSPATA6 and miR-182, RIP assay was conducted using antibody Ago2, a key component of RISC complex. Consistent with bioinformatics analysis results and dual-luciferase reporter assay, RIP assay demonstrated that circSPATA6 and miR-182 were notably enriched in the Anti-Ago2 

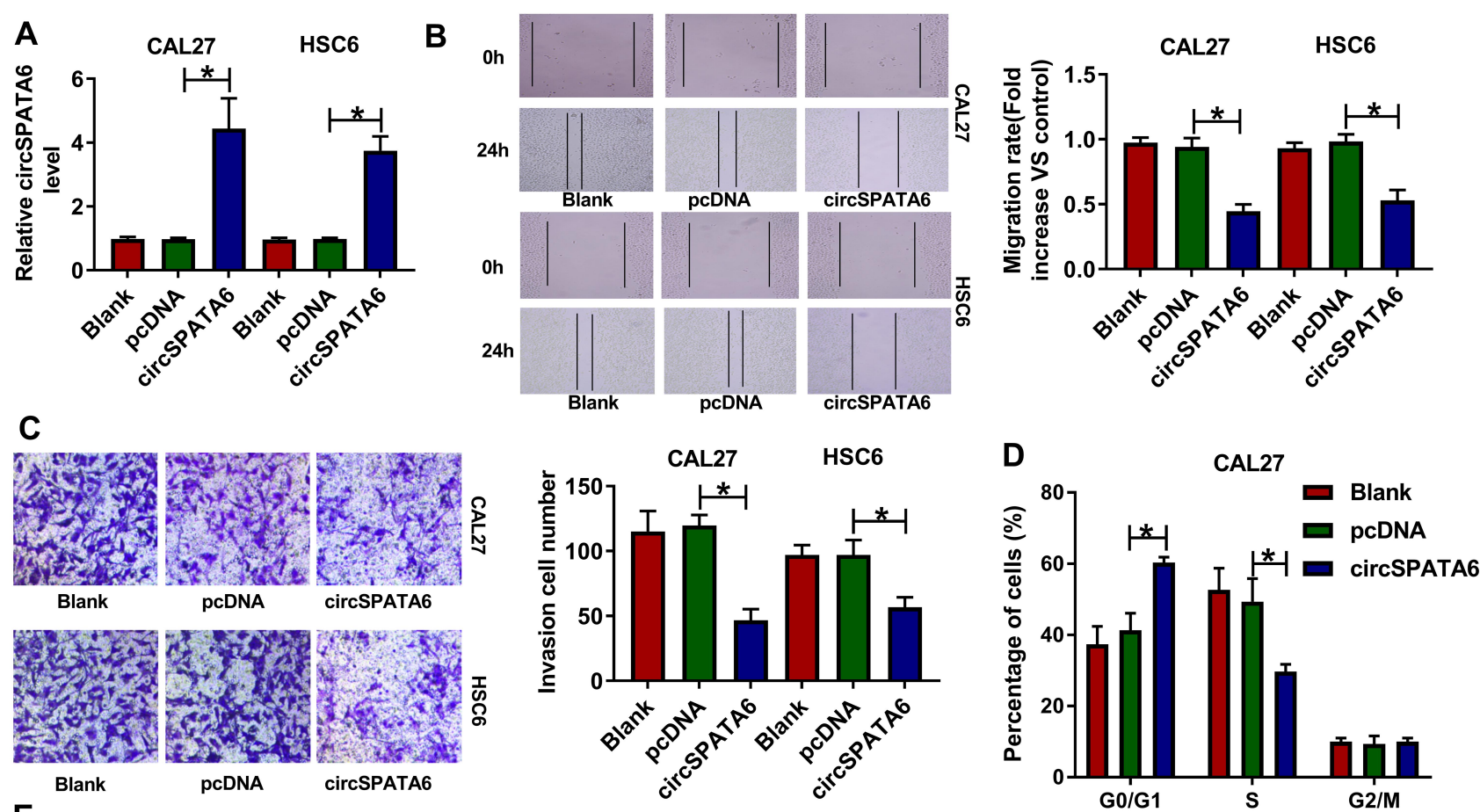

E

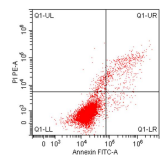

Blank

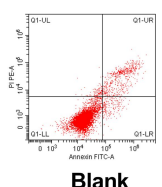

Blank

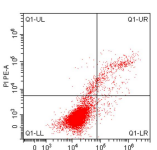

pcDNA
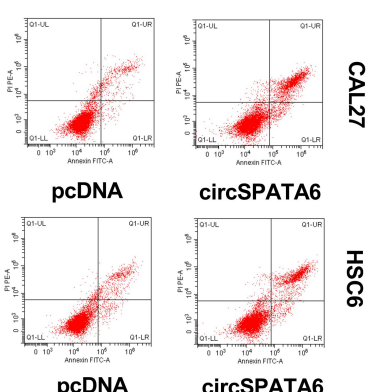

circSPATA6

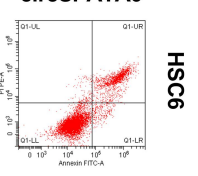

circSPATA6
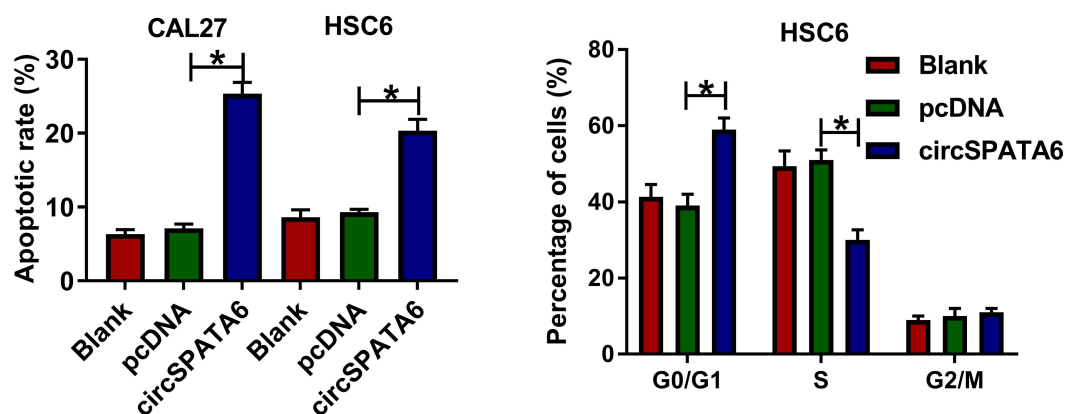

Figure 2 CircSPATA6 overexpression suppressed migration, invasion, cell cycle, and promoted apoptosis of OSCC cells. CAL-27 and HSC6 cells were transfected with Blank, pcDNA, and circSPATA6. (A) The expression level of circSPATA6 was examined in transfected CAL-27 and HSC6 cells. (B) Wound healing assay was applied to detect migration rate in transfected CAL-27 and HSC6 cells. (C) Transwell assay was performed to assess invasion in transfected CAL-27 and HSC6 cells. (D and E) Flow cytometry assays were conducted to examine cell cycle distribution and apoptosis rate in transfected CAL-27 and HSC6 cells. *P <0.05.

group compared to the Anti-IgG control group (Figure 3D and E). Also, RT-qPCR results verified that miR-182 was decreased in OSCC cell lines (CAL-27, HSC6, UM1, and SCC9) relative to $\mathrm{HOK}$ cells (Figure 3F). Whereafter, we detected the expression level of miR-182 in pcDNAcircSPATA6 or si-circSPATA6-transfected CAL-27 and HSC6 cells. As presented in Figure 3G and H, the manipulation of circSPATA6 expression could change the expression level of miR-182, showing that the expression level of miR-182 was decreased in OSCC cells transfected with circSPATA6, and miR-182 level was increased in OSCC cells transfected with si-circSPATA6, when compared with their control groups. Overall, these data discovered that circSPATA6 interacts with miR-182 to block its expression.

\section{TRAF6 Served as a Target of miR-I 82}

Next, the latent target mRNA of miR-182 was predicted by bioinformatics software DIANA TOOL. As a result, there were some complementary sites between TRAF6-3'UTR and miR182 (Figure 4A), as proved by the dual-luciferase reporter assay. Data showed that miR-182 mimic significantly reduced the luciferase activity of TRAF6-WT reporter vector but not that of TRAF6-MUT reporter vector (Figure 4B and C). Also, the results of RIP assay indicated that both miR-182 and TRAF6 were enriched in the anti-Ago2 group compared with the ant-IgG control group (Figure 4D and E). Synchronously, the significant downregulation of TRAF6 was observed in OSCC cell lines (CAL-27, HSC6, UM1, and SCC9) relative to HOK cells (Figure 4F). Moreover, we further verified that 
A

circSPATA6 $-\mathrm{WT}\left(5^{\prime} \ldots 3^{\prime}\right)$

$\operatorname{miR}-182\left(3^{\prime} \ldots 5^{\prime}\right)$

circSPATA6-MUT( $\left.5^{\prime} . . .3^{\prime}\right)$
GCAGCACCCUCAACAAUGCCAAAG |l|||||

UCACACUCAAGAUGGUAACGGUUU

GCAGCACCCUCAACAAGUAACCGG

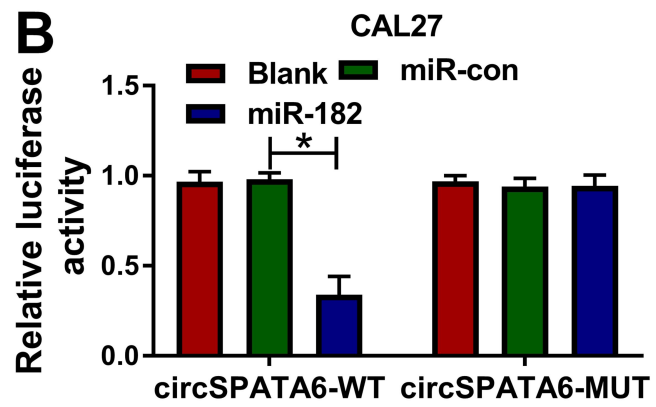

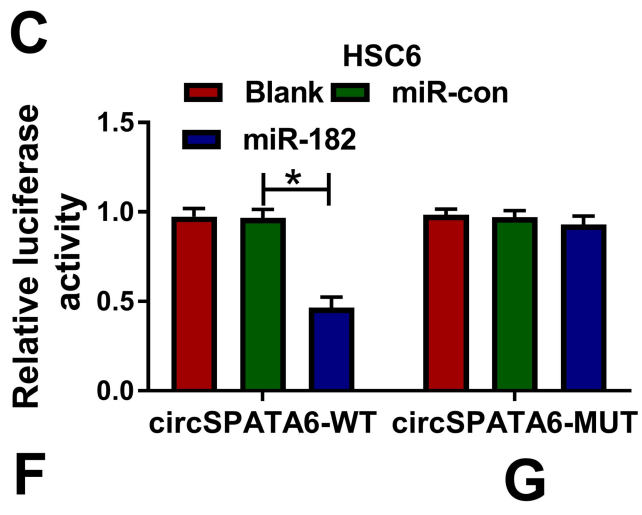

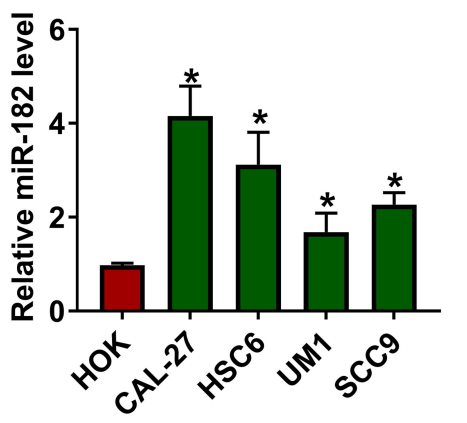

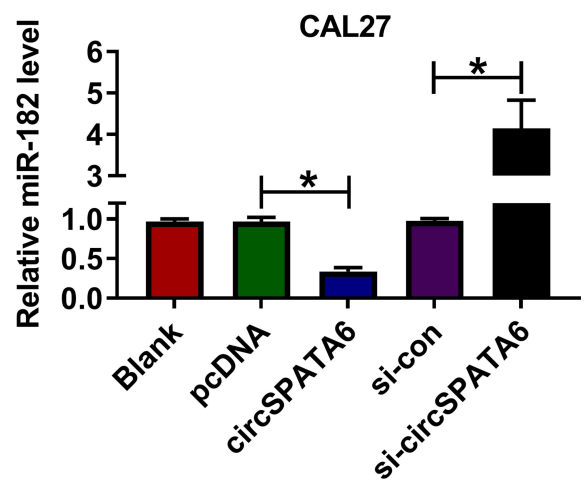

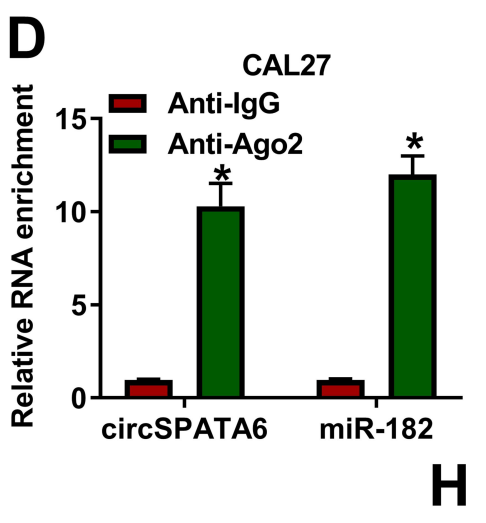

$\mathrm{H}$
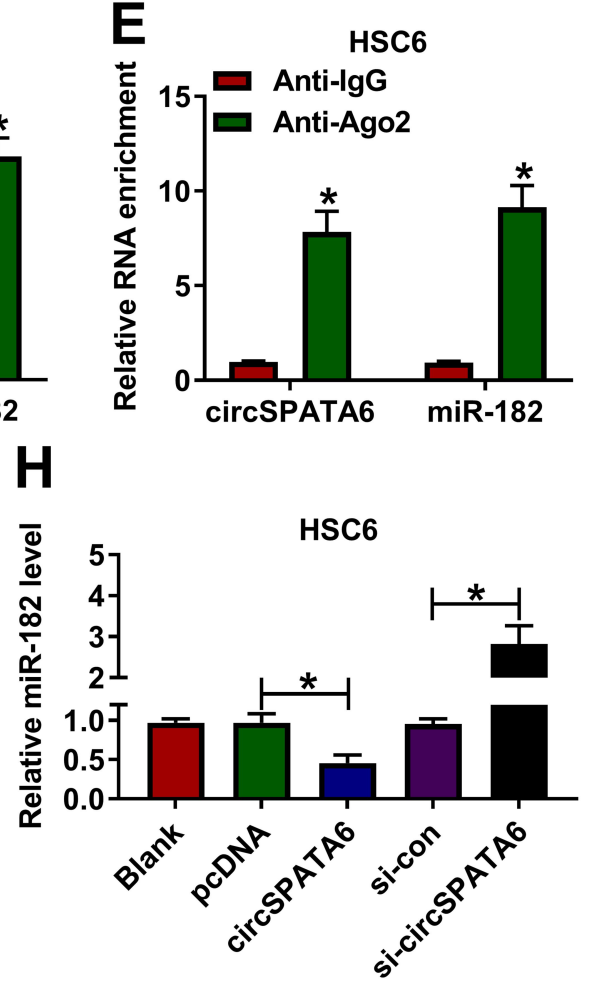

Figure 3 MiR-182 was a direct target of circSPATA6. (A) Circular RNA interactome was employed to predicate the interaction between circSPATA6 and miR-182. (B and C) The underlying relationship was verified using dual-luciferase reporter assay. (D and E) RIP assay was conducted in CAL-27 and HSC6 cell extracts to examine miR-I82 endogenously associated with circSPATA6. (F) Relative miR-182 level was detected in HOK, CAL-27, HSC6, UMI, and SCC9 cells. (G and H) MiR-I82 level was examined in CAL-27 and HSC6 cells transfected with Blank, pcDNA, circSPATA6, si-con, si-circSPATA6. $* P<0.05$.

miR-182 mimics hindered the protein level of TRAF6, and miR-182 inhibitor expedited TRAF6 protein level in CAL-27 and HSC6 cells (Figure 4G and H). Taken together, TRAF6 was a target of miR-182 in OSCC cells.

\section{Overexpression of miR-182 Partly Abrogated the Effect of circSPATA6 on Migration, Invasion, Cell Cycle Progression, and Apoptosis in OSCC Cells}

As mentioned above, circSPATA6 played a crucial role in the progression of OSCC cells, and miR-182 was a direct target of circSPATA6. Thus, we performed rescue experiments to explore whether circSPATA6 could regulate OSCC progression by targeting miR-182. As shown in Figure 5A and B, the upregulation of circSPATA6 restrained miR-182 expression level, which was relieved by the introduction of miR-182 mimics in CAL-27 and HSC6 cells. Functionally, the miR-182 overexpression partly reversed the suppressive action of pcDNA-circSPATA6 on migration (Figure 5C and D) and invasion (Figure 5E and F) in CAL-27 and HSC6 cells. Moreover, flow cytometry analysis indicated that the miR-182 upregulation apparently overturned pcDNAcircSPATA6-induced increase in cell cycle arrest (Figure $5 \mathrm{G}$ and $\mathrm{H}$ ) and apoptotic rate (Figure 5I and $\mathrm{J}$ ) in CAL-27 and HSC6 cells. In a word, circSPATA6 could constrain OSCC cells' progression partially through modulating miR-182. 

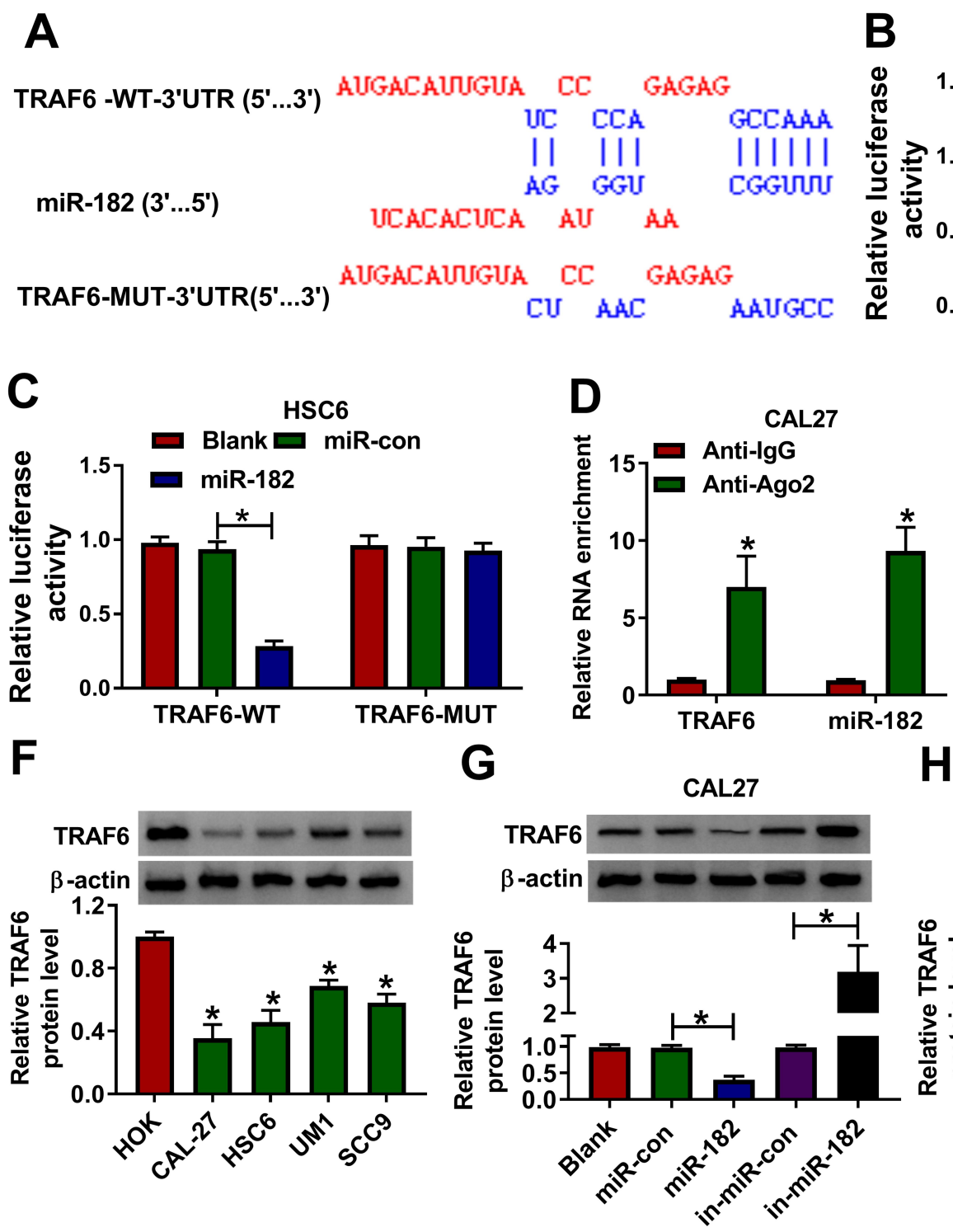
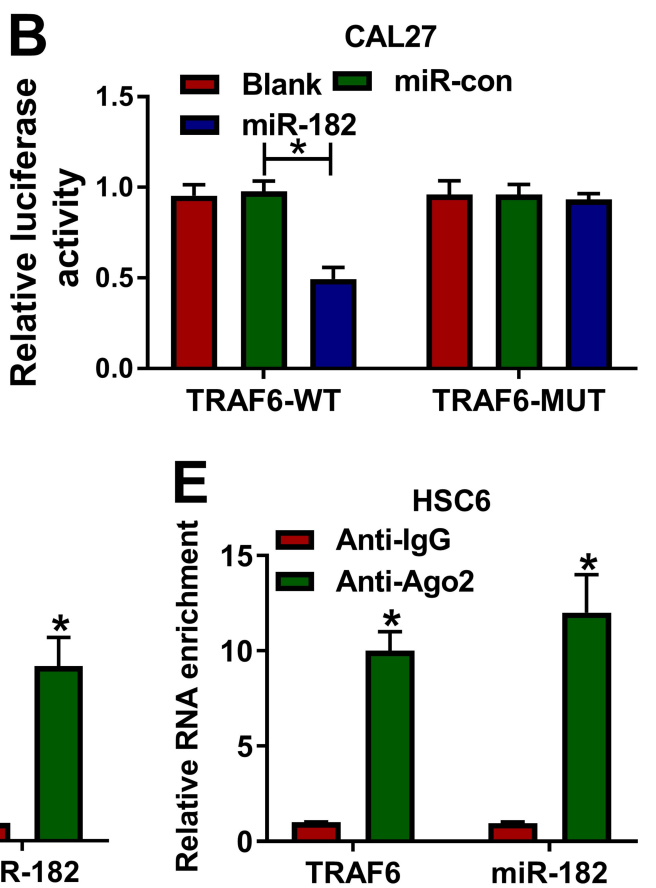

$\mathrm{H}$

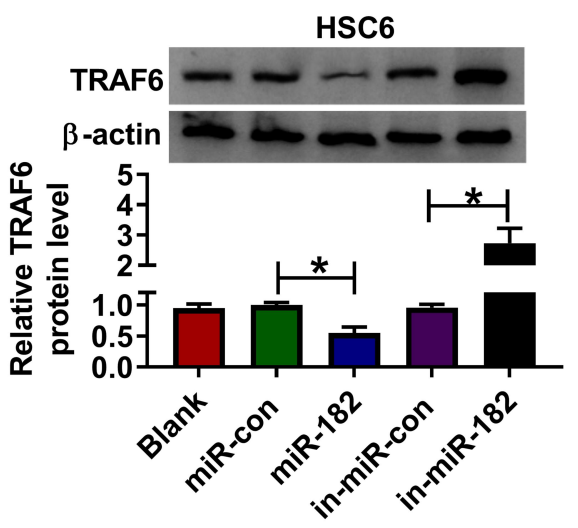

Figure 4 MiR-182 directly bound with TRAF6. (A) Putative binding sequences between miR-182 and TRAF6-3'UTR, and mutant sites in TRAF6-3'UTR reporter. (B and C) Dual-luciferase reporter assays were applied to verify the prediction between miR-182 and TRAF6-3'UTR. (D and E) The interaction between miR-I82 and TRAF6-3'UTR was proved by RIP assays. (F) Western blot assay was conducted to detect the protein level of TRAF6 in HOK, CAL-27, HSC6, UMI, and SCC9 cells. (G and H) TRAF6 protein level was assessed in CAL-27 and HSC6 cells transfected with Blank, miR-con, miR-182, in-miR-con, and in-miR-182. $* P<0.05$.

TRAF6 Silencing Abrogated miR-I 82

Knockdown-Mediated Migration, Invasion, Cell Cycle Progression, and Apoptosis in OSCC Cells

Then, to investigate the regulatory mechanism of miR-182 in OSCC cells, we conducted the rescue assays. Western blot results presented that the downregulation of miR-182 accelerated TRAF6 protein level, while si-TRAF6 abated the effect in CAL-27 and HSC6 cells (Figure 6A and B). Moreover, the capacities of migration (Figure $6 \mathrm{C}$ and $\mathrm{D}$ ) and invasion (Figure $6 \mathrm{E}$ and $\mathrm{F}$ ) were impaired by in-miR -182 and then partly abolished by the deficiency of TRAF6 in CAL-27 and HSC6 cells. Meanwhile, the si-TRAF6 partly overturned the positive action of miR-182 knockdown on cell cycle arrest (Figure 6G and $\mathrm{H}$ ) and apoptotic rate (Figure 6I and J) in CAL-27 and HSC6 cells. Collectively, miR-182 downregulation repressed the progression of OSCC cells by targeting TRAF6.

\section{CircSPATA6 Inhibited OSCC Cell}

\section{Growth in vivo}

Additionally, a xenograft tumor mouse model was established to explore the impact of circSPATA6 on tumor growth. As displayed in Figure 7A and B, tumor volume 


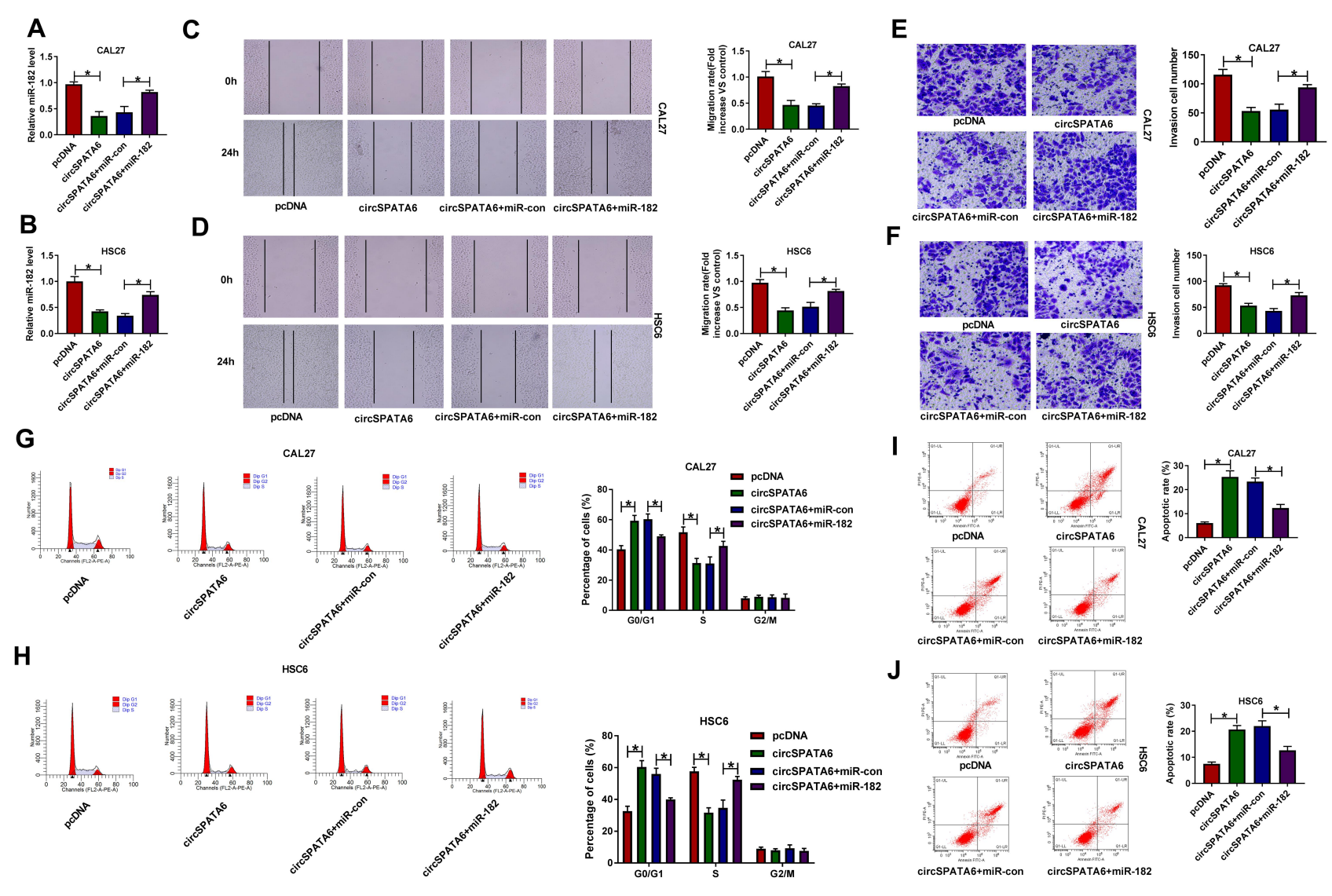

Figure 5 Overexpression of miR-182 overturned the inhibitory effect of circSPATA6 on OSCC progression. CAL-27 and HSC6 cells were transfected with pcDNA, circSPATA6, circSPATA6 + miR-con, and circSPATA6 + miR-182. (A and B) Relative miR-182 level was measured in transfected CAL-27 and HSC6 cells. (C and D) Migration ability was examined in transfected CAL-27 and HSC6 cells. (E and F) Invasion capacity was detected in transfected CAL-27 and HSC6 cells. (G and H) Cell cycle distribution was determined in transfected CAL-27 and HSC6 cells. (I and J) Apoptosis rate was assessed in transfected CAL-27 and HSC6 cells. $* P<0.05$.

and weight were declined in presence of circSPATA6 upregulation, verifying the suppression action of circSPATA6 on OSCC cell growth in vivo. Apart from that, the levels of circSPATA6 (Figure 7C) and TRAF6 (Figure 7E) were increased, and miR-182 level (Figure 7D) was reduced in tumor tissues from the circSPATA6 group relative to the Blank and pcDNA group. These data suggested that circSPATA6 overexpression impeded the tumor growth in OSCC through regulating the miR-182/ TRAF6 axis.

\section{Exosomal circSPATA6 Curbed OSCC Cell Growth in vitro}

Given that circSPATA6 exhibited lower expression in OSCC serum, we investigated whether exosomal circSPATA6 could affect the tumor growth in vitro. Firstly, exosomes from OSCC cells were isolated by ultracentrifugation, and then examined by a transmission electron microscope. As showed in Figure 8A, exosomes were typical rounded particles. Then, exosomes isolated from
pcDNA or circSPATA6-transfected CAL-27 and HSC6 cells were co-incubated with these transfected CAL-27 and HSC6 cells, respectively. Data suggested that circSPATA6 level was obviously increased in the CAL27/HSC6-circSPATA6-exosome group in comparison with the CAL-27/HSC6-pcDNA-exosome and control groups (Figure $8 \mathrm{~B}$ and $\mathrm{C}$ ). Furthermore, functional analysis revealed that the introduction of exosomes from transfected CAL-27 and HSC6 cells reduced migration (Figure 8D and E) and invasion (Figure 8F and G), and enhanced cell cycle arrest (Figure $8 \mathrm{H}$ and I) and apoptotic rate (Figure $8 \mathrm{~J}$ and $\mathrm{K}$ ) in transfected CAL-27 and HSC6 cells, when compared with their respective control groups. Together, these data indicated that the exosomal circSPATA6 could hinder tumor growth in OSCC cells.

\section{Discussion}

Up to now, a growing number of circRNAs were increasingly identified owing to the development of the highthroughput sequencing. ${ }^{33,34}$ It has been acknowledged 


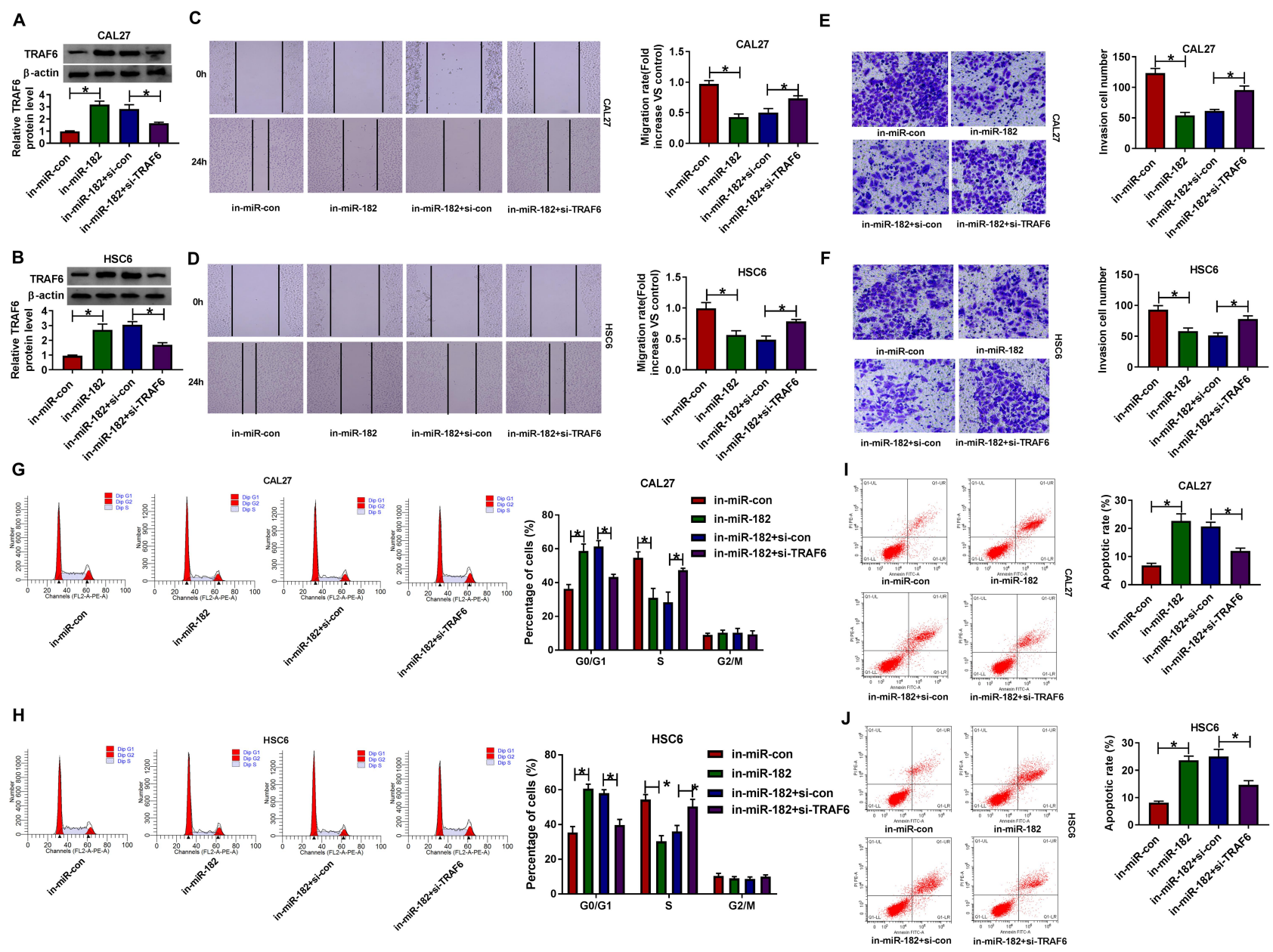

Figure 6 The suppression action of miR-182 knockdown on OSCC progression was reversed by the downregulation of TRAF6. CAL-27 and HSC6 cells were transfected with in-miR-con, in-miR-182, in-miR-182+si-con, and in-miR-182+si- TRAF6. (A and B) TRAF6 protein level was detected in transfected CAL-27 and HSC6 cells. (C and D) Migration rate was detected in transfected CAL-27 and HSC6 cells. (E and F) Invasion was assessed in transfected CAL-27 and HSC6 cells. (G and H) Percentage of cells was measured in transfected CAL-27 and HSC6 cells. ( $\mathbf{I}$ and J) Apoptosis rate was analyzed in transfected CAL-27 and HSC6 cells. $* P<0.05$.

that circRNAs could act as a potential attractive biomarker due to the stability and resistance to RNase activity compared with linear RNA. ${ }^{35}$ Accumulating evidence suggested that the dysregulation of circRNAs participated in the progression and development of multiple cancers, including OSCC. In this paper, circSPATA6, located on chromosome 1 , has been verified to be the low expression in OSCC tissues and cell lines, in agreement with the previous report. ${ }^{13}$ Due to the diagnostic value and the stable loop structure, circSPATA6 can be used as a potential biomarker for the prognosis of OSCC. Functionally, circSPATA6 inhibited OSCC cells migration and invasion, and boosted cell cycle arrest and apoptosis in vitro. Moreover, the suppression effect of circSPATA6 on OSCC tumor growth was verified in vivo. These results validated that circSPATA6 might serve as a tumor suppressor in OSCC.
Currently, one hypothesis proposed that circRNAs could work as a ceRNA for miRNAs to release miRNAtargeted mRNAs expression. ${ }^{36,37}$ Thus, in OSCC cells, we explored whether circSPATA6 could exert the role in a miRNA-mRNA dependent manner. In this manuscript, miR-182 as a direct target of circSPATA6 was predicted, as proved by the dual-luciferase reporter and RIP assays. Meanwhile, the high expression of miR-182 was viewed in OSCC cell lines. Importantly, our data presented that the overexpression of miR-182 could partially reverse the circSPATA6-mediated decrease in migration and invasion, and the increase in cell cycle arrest and apoptosis in OSCC cells. Consistent with our results, the inductive effect of miR-182 on cell growth and metastasis was also demonstrated in OSCC. ${ }^{23,24}$ It has been acknowledged that miRNAs could modulate the formation and development of tumors through target genes. In this study, our data 


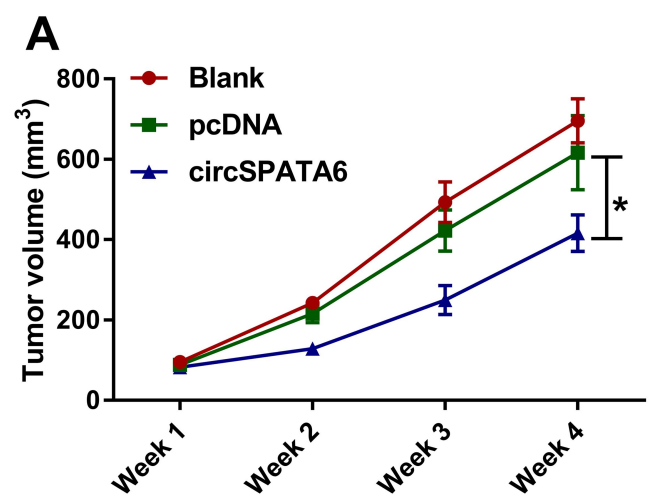

C

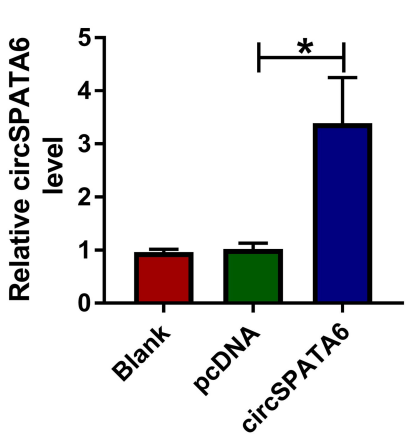

D

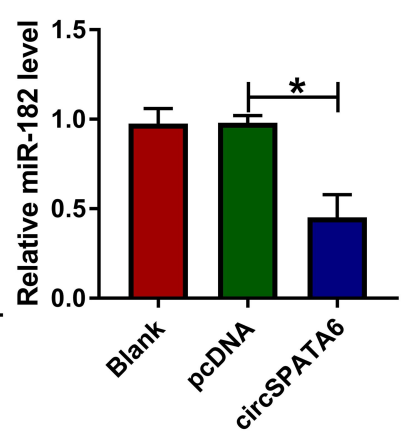

B
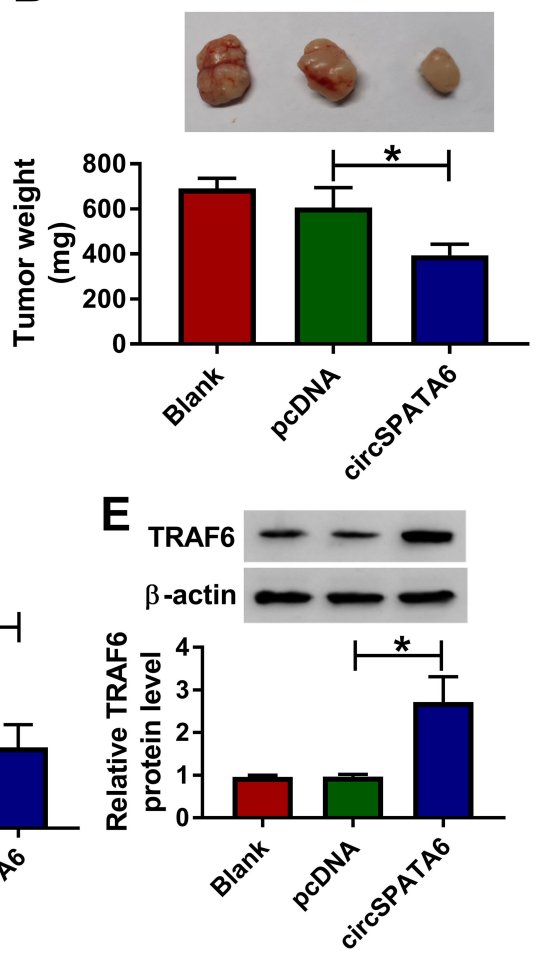

Figure 7 CircSPATA6 repressed tumor growth in OSCC in vivo. CAL-27 cells transfected with Blank, pcDNA, and circSPATA6 were subcutaneously injected into the nude mice. (A) Growth curve of the xenografted tumor. (B) Weights of resected tumor masses. (C and D) The mRNA levels of circSPATA6 and miR-182 in xenografts were measured. (E) TRAF6 protein level was examined. $* P<0.05$.
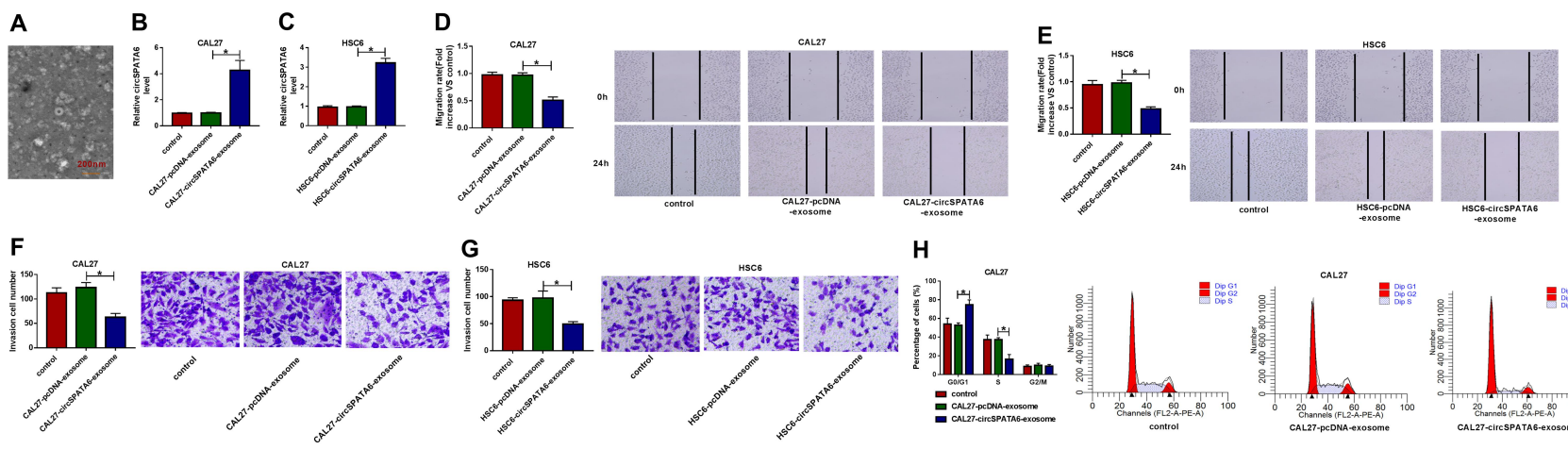

H
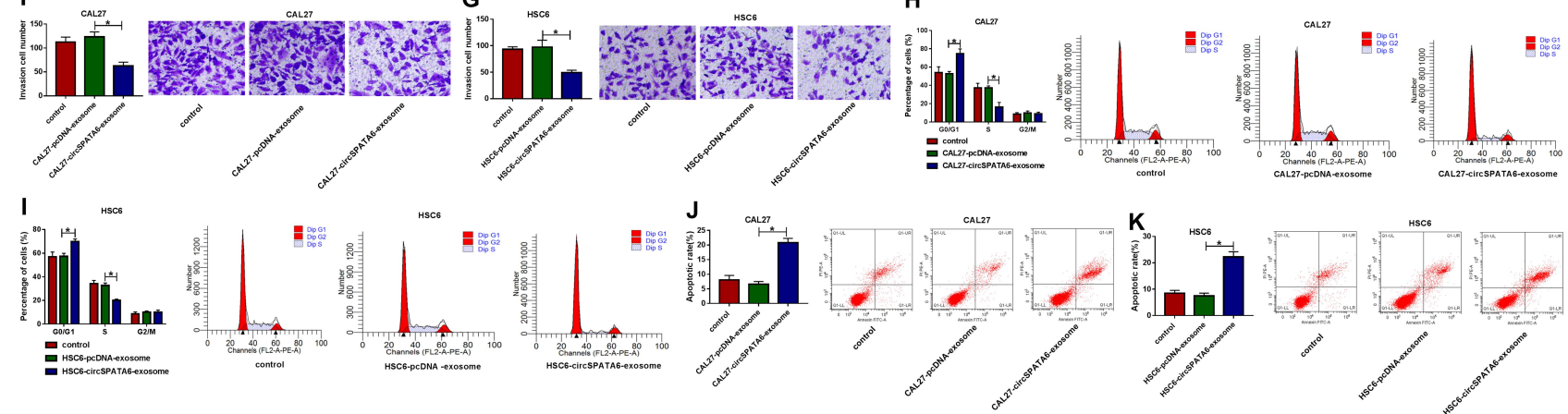

Figure 8 Exosomal circSPATA6 inhibited the growth of OSCC cells. (A) A transmission electron microscope was used to detect the morphology of exosomes. (B-K) exosomes were isolated from CAL-27 and HSC6 cells transfected with pcDNA or circSPATA6, and then, co-cultured with transfected CAL-27 and HSC6, respectively. (B and $\mathbf{C}$ ) Relative circSPATA6 level was detected in exosomes from un-transfected or transfected CAL-27 or HSC6 cells. (D and E) Migration rate was measured in exosomes from un-transfected or transfected CAL-27 or HSC6 cells. (F and $\mathbf{G})$ Invasion cell number was examined in exosomes from un-transfected or transfected CAL-27 or HSC6 cells. ( $\mathbf{H}$ and $\mathbf{I})$ The percentage of cells was analyzed in exosomes from un-transfected or transfected CAL-27 or HSC6 cells. (J and K) Apoptosis rate was assessed in exosomes from un-transfected or transfected CAL-27 or HSC6 cells. $* P<0.05$. 
proved that miR-182 interacted with TRAF6 to repress its expression. TRAF6 has been pointed out to be implicated in the oncogenicity in oral carcinoma. ${ }^{29}$ Moreover, TRAF6 was previously proved to promote tumorigenesis of human oral cancer cells and oral tumor growth in OSCC. ${ }^{38}$ Hence, we choose TRAF6 for further research. In this paper, TRAF6 was found to be decreased in OSCC cells, consistent with the earlier work. ${ }^{30}$ Furthermore, downregulation of TRAF6 abolished the inhibitory effect of miR-182 knockdown on the progression of OSCC cells, further supporting the regulatory function of circSPATA6 on OSCC progression was mediated in part by the miR-182/ TRAF6 axis. In addition, TRAF6 has been reported to be involved in the activation of $\mathrm{NF}-\kappa \mathrm{B}$ and Akt signaling pathway in the treatment of oral cancer. ${ }^{38}$ Therefore, we would explore the role of the circSPATA6/miR-182/ TRAF6 axis on these signaling pathways in future studies.

Additionally, previous studies have displayed that circRNAs were enriched and stable in exosomes. ${ }^{39,40}$ Moreover, some studies suggested that exosomal circRNAs, a novel biomarker, were relevant to the tumorigenesis in various cancers. $^{41-43}$ In this research, we observed that circSPATA6 was decreased in OSCC cellderived exosomes. Functionally, exosomal circSPATA6 was first verified to hinder the progression of OSCC cells. Therefore, in future research, we will focus on exploring whether exosomal circSPATA6 can regulate OSCC tumor progression through modulating the miR-182/TRAF6 axis.

\section{Conclusion}

In this study, our data revealed that circSPATA6 could as a sponge of miR-182 to increase TRAF6 expression, thereby boosting the progression of OSCC. These findings provided a novel therapeutic target for OSCC.

\section{Data Sharing Statement}

The analyzed data sets generated during the present study are available from the corresponding author on reasonable request.

\section{Ethics Approval and Consent to Participate}

The present study was approved by the ethical review committee of Inner Mongolia Baotou Steel Hospital. Written informed consent was obtained from all enrolled patients.

Patients agree to participate in this work.

\section{Funding}

No funding was received.

\section{Disclosure}

The authors declare that they have no conflicts of interest for this work.

\section{References}

1. Jayaraj R, Kumarasamy C, Madurantakam Royam M, Devi A, Baxi S. Letter to the editor: is HIF-1 $\alpha$ a viable prognostic indicator in OSCC? A critical review of a meta-analysis study. World J Surg Oncol. 2018;16(1):111. doi:10.1186/s12957-018-1408-4

2. Siegel RL, Miller KD. Cancer Statistics, 2019. CA. 2019;69(1):7-34.

3. Miller KD, Nogueira L, Mariotto AB, Rowland JH, Yabroff KR, Alfano CM. Cancer treatment and survivorship statistics, 2019. Cancer J Clin. 2019;69(5):363-385. doi:10.3322/caac.21565

4. Kristensen LS, Andersen MS, Stagsted LVW, Ebbesen KK, Hansen TB. The biogenesis, biology and characterization of circular RNAs. Int J Med. 2019;20(11):675-691.

5. Li X, Yang L, Chen LL, Biogenesis T. Functions, and Challenges of Circular RNAs. Mol Cell. 2018;71(3):428-442.

6. Zhang HD, Jiang LH, Sun DW, Hou JC, Ji ZL. CircRNA: a novel type of biomarker for cancer. Breast Cancer. 2018;25(1):1-7.

7. Xia L, Song M, Sun M, Wang F, Yang C. Circular RNAs as Biomarkers for Cancer. Adv Exp Med Biol. 2018;1087:171-187.

8. Li Y, Zheng Q, Bao C, et al. Circular RNA is enriched and stable in exosomes: a promising biomarker for cancer diagnosis. Cell Res. 2015;25(8):981-984.

9. Li T, Shao Y, Fu L, et al. Plasma circular RNA profiling of patients with gastric cancer and their droplet digital RT-PCR detection. $J$ Mol Med (Berl). 2018;96(1):85-96.

10. Liang G, Yang Y, Niu G, Tang Z, Li K. Genome-wide profiling of Sus scrofa circular RNAs across nine organs and three developmental stages. DNA Res. 2017;24(5):523-535. doi:10.1093/dnares/dsx022

11. Ouyang SB, Wang J, Zhao SY, Zhang XH, Liao L. CircRNA_0109291 regulates cell growth and migration in oral squamous cell carcinoma and its clinical significance. Iran J Basic Med Sci. 2018;21(11):1186-1191. doi:10.22038/IJBMS.2018.30347.7313

12. Gao L, Zhao C, Li S, et al. circ-PKD2 inhibits carcinogenesis via the miR-204-3p/APC2 axis in oral squamous cell carcinoma. Mol Carcinog. 2019;58(10):1783-1794. doi:10.1002/mc.23065

13. Wang YF, Li BW, Sun S, et al. Circular RNA expression in oral squamous cell carcinoma. Front Oncol. 2018;8:398. doi:10.3389/ fonc.2018.00398

14. Bartel DP. MicroRNAs: genomics, biogenesis, mechanism, and function. Cell. 2004;116(2):281-297. doi:10.1016/s0092-8674(04) 00045-5

15. Li T, Li M, Xu C, et al. miR-146a regulates the function of Th17 cell differentiation to modulate cervical cancer cell growth and apoptosis through NF-KB signaling by targeting TRAF6. Oncol Rep. 2019;41 (5):2897-2908. doi:10.3892/or.2019.7046

16. Xu Q, Zhu Q, Zhou Z, et al. MicroRNA-876-5p inhibits epithelial-mesenchymal transition and metastasis of hepatocellular carcinoma by targeting BCL6 corepressor like 1. Biomed Pharmacother. 2018;103:645-652. doi:10.1016/j.biopha.2018.04.037

17. Hou G, Xu W, Jin Y, Wu J, Pan Y, Zhou F. MiRNA-217 accelerates the proliferation and migration of bladder cancer via inhibiting KMT2D. Biochem Biophys Res Commun. 2019;519(4):747-753. doi:10.1016/j.bbrc.2019.09.029

18. Zha JF, Chen DX. MiR-655-3p inhibited proliferation and migration of ovarian cancer cells by targeting RAB1A. Eur Rev Med Pharmacol Sci. 2019;23(9):3627-3634. 
19. Wang N, Cao QX, Tian J. Circular RNA MTO1 inhibits the proliferation and invasion of ovarian cancer cells through the miR-182-5p/ KLF15 axis. Int $J$ oncol. 2020;29:963689720943613.

20. Mao Y, Lv M, Cao W, et al. Circular RNA 000554 represses epithelial-mesenchymal transition in breast cancer by regulating microRNA-182/ZFP36 axis. FASEB J. 2020;34(9):11405-11420. doi:10.1096/fj.201903047R

21. Liang L, Li L. Down-regulation of circNRIP1 promotes the apoptosis and inhibits the migration and invasion of gastric cancer cells by miR-182/ROCK1 axis. Onco Targets Ther. 2020;13:6279-6288. doi:10.2147/OTT.S221633

22. Wang L, Jiang $\mathrm{H}$, Li W, et al. Overexpression of TP53 mutation-associated microRNA-182 promotes tumor cell proliferation and migration in head and neck squamous cell carcinoma. Arch Oral Biol. 2017;73:105-112. doi:10.1016/j.archoralbio.2016.09.012

23. Guo Y, Qiao X, Zhu L, Song R. MIR-182-5p modulates oral squamous cell carcinoma migration and invasion via targeting MTSS1 gene. Pathol Oncol Res. 2020;26(2):1007-1013. doi:10.1007/s12253019-00647-8

24. Li N, Nan CC, Zhong XY, et al. miR-182-5p promotes growth in oral squamous cell carcinoma by inhibiting CAMK2N1. Cell Physiol Biochem. 2018;49(4):1329-1341. doi:10.1159/000493411

25. Zhang X, Guo Y, Xu X, et al. miR-146a promotes Borna disease virus 1 replication through IRAK1/TRAF6/NF- $\kappa$ B signaling pathway. Virus Res. 2019;271:197671. doi:10.1016/j.virusres.2019.197671

26. Wei J, Yuan Y, Jin C, et al. The ubiquitin ligase TRAF6 negatively regulates the JAK-STAT signaling pathway by binding to STAT3 and mediating its ubiquitination. PLoS One. 2012;7(11):e49567. doi:10.1371/journal.pone.0049567

27. Hu Q, Song J, Ding B, Cui Y, Liang J, Han S. miR-146a promotes cervical cancer cell viability via targeting IRAK1 and TRAF6. Oncol Rep. 2018;39(6):3015-3024. doi:10.3892/or.2018.6391

28. Wu H, Lu XX, Wang JR, et al. TRAF6 inhibits colorectal cancer metastasis through regulating selective autophagic CTNNB1/ $\beta$ catenin degradation and is targeted for GSK3B/GSK3 $\beta$-mediated phosphorylation and degradation. Autophagy. 2019;15 (9):1506-1522. doi:10.1080/15548627.2019.1586250

29. Hung PS, Liu CJ, Chou CS, et al. miR-146a enhances the oncogenicity of oral carcinoma by concomitant targeting of the IRAK1, TRAF6 and NUMB genes. PLoS One. 2013;8(11):e79926. doi:10.1371/journal.pone.0079926

30. Min SK, Jung SY, Kang HK, et al. Functional diversity of miR-146a$5 \mathrm{p}$ and TRAF6 in normal and oral cancer cells. Int J Oncol. 2017;51 (5):1541-1552. doi:10.3892/ijo.2017.4124
31. Piao HY, Guo S, Wang Y, Zhang J. Exosomal long non-coding RNA CEBPA-AS1 inhibits tumor apoptosis and functions as a non-invasive biomarker for diagnosis of gastric cancer. OncoTargets Therapy. 2020;13:1365-1374. doi:10.2147/OTT.S238706

32. Militello G, Weirick T, John D, Döring C, Dimmeler S, Uchida S. Screening and validation of lncRNAs and circRNAs as miRNA sponges. Brief Bioinform. 2017;18(5):780-788. doi:10.1093/bib/ bbw053

33. Yang C, Wei Y, Yu L, Xiao Y. Identification of altered circular RNA expression in serum exosomes from patients with papillary thyroid carcinoma by high-throughput sequencing. Med Sci Monit. 2019;25:2785-2791. doi:10.12659/MSM.915658

34. Qiu X, Ke X, Ma H, et al. Profiling and bioinformatics analyses reveal differential expression of circular RNA in tongue cancer revealed by high-throughput sequencing. J Cell Biochem. 2019;120 (3):4102-4112. doi:10.1002/jcb.27695

35. Jeck WR, Sharpless NE. Detecting and characterizing circular RNAs. Nat Biotechnol. 2014;32(5):453-461. doi:10.1038/nbt.2890

36. Hansen TB, Jensen TI, Clausen BH, et al. Natural RNA circles function as efficient microRNA sponges. Nature. 2013;495 (7441):384-388. doi:10.1038/nature11993

37. Zhong Y, Du Y, Yang X, et al. Circular RNAs function as ceRNAs to regulate and control human cancer progression. Mol Cancer. 2018;17 (1):79. doi:10.1186/s12943-018-0827-8

38. Wu YH, Wu WS, Liu LC, et al. Bortezomib enhances radiosensitivity in oral cancer through inducing autophagy-mediated TRAF6 oncoprotein degradation. J Exp Clin Cancer Res. 2018;37(1):91. doi:10.1186/s13046-018-0760-0

39. Fanale D, Taverna S, Russo A, Bazan V. Circular RNA in Exosomes. Adv Exp Med Biol. 2018;1087:109-117.

40. Hou J, Jiang W, Zhu L, et al. Circular RNAs and exosomes in cancer: a mysterious connection. J Oncol. 2018;20(9):1109-1116.

41. Zhang X, Wang S, Wang H, et al. Circular RNA circNRIP1 acts as a microRNA-149-5p sponge to promote gastric cancer progression via the AKT1/mTOR pathway. Int J Oncol. 2019;18(1):20.

42. Li Z, Yanfang W, Li J, et al. Tumor-released exosomal circular RNA PDE8A promotes invasive growth via the miR-338/MACC1/MET pathway in pancreatic cancer. Cancer Lett. 2018;432:237-250.

43. Chen W, Quan Y, Fan S, et al. Exosome-transmitted circular RNA hsa_circ_0051443 suppresses hepatocellular carcinoma progression. Cancer Lett. 2020;475:119-128.
Cancer Management and Research

\section{Publish your work in this journal}

Cancer Management and Research is an international, peer-reviewed open access journal focusing on cancer research and the optimal use of preventative and integrated treatment interventions to achieve improved outcomes, enhanced survival and quality of life for the cancer patient.
Dovepress

The manuscript management system is completely online and includes a very quick and fair peer-review system, which is all easy to use. Visit http://www.dovepress.com/testimonials.php to read real quotes from published authors. 